\title{
Radon emission rate and analysis of its influencing parameters
}

\author{
Thomas Neugebauer, \\ Hans Hingmann, \\ Jonas Buermeyer, \\ Volker Grimm, \\ Joachim Breckow
}

\begin{abstract}
The geological and structural conditions define the radon situation inside a building. While the geological realities can be specified by the content of radium- 226 and the ratio of radon-222 emitted from the ground the structural conditions are defined by the tightness of the building envelope. The radon concentration inside has an unsteady character, which is caused by meteorological conditions outside and the air change rate (ACH or ACR), which in turn is influenced by the residents' behaviour such as venting and heating. For the assessment of the radon exposition, it is necessary to perform measurements for a long time. An approach to reduce this time by eliminating the inhabitants influence on the radon concentration is the radon emission rate, also known as radon entry rate. This variable is based on the measurement of the radon concentration and the parallel determination of the air change rate via a tracer gas method, the result expresses a released activity per time. Due to their noisy character, it is necessary to apply a smoothing algorithm to the input parameters. In addition to mean values, the use of window functions, known from digital signal processing, was analysed. For the verification of the whole calculation procedure, simulations and measurements under defined conditions were used. Furthermore, measurements in an uninhabited house showed proof of the capability of the assessment of the radon potential. First examinations of influencing parameters of the radon emission rate showed a possible dependence on the temperature difference inside and outside the building.
\end{abstract}

Key words: air change rate $\bullet$ radon emission rate $\bullet$ radon entry rate $\bullet$ radon exposition $\bullet$ window function

T. Neugebauer, H. Hingmann, J. Buermeyer, V. Grimm ${ }^{\bowtie}$, J. Breckow

Technische Hochschule Mittelhessen (THM), Institut für Medizinische Physik und Strahlenschutz (IMPS),

Wiesenstrasse 14, 35394 Giessen, Germany,

Tel.: +49 641309 2317, Fax: +49 641309 2973,

E-mail: volker.grimm@mni.thm.de

Received: 15 December 2015

Accepted: 24 March 2016

\section{Introduction}

Radon ( $\mathrm{Rn}-222)$ is a radioactive noble gas. It is a part of the uranium-radium-series and originates from its parent nuclide radium-226 which is contained in the earth's crust. Due to emanation, transportation processes and exhalation it reaches the atmosphere and can enter a building by different pathways (e.g. cracks in the bottom plate or leaky cable ducts) where it can be enriched to high concentrations. Many epidemiological studies showed a correlation between exposure to radon and the occurrence of lung cancer [1-3]. This is the reason the European Union (EU) attached importance to radon when preparing the Council Directive 2013/59/Euratom [4].

This directive has to be implemented into national law of the EU member-states by February 2018, and it reveals the first steps to deal with the general radon exposition. A reference level of an annual average radon concentration of $300 \mathrm{~Bq} \cdot \mathrm{m}^{-3}$ for dwellings and workplaces is introduced and the member states are ordered to develop 'radon action plans'. Amongst the requirements of the radon action plans, the development of methods and indicators to measure and evaluate the radon concentrations are expected. 
Measurements of the radon soil gas concentration and permeability can give an overview of the geogenic radon potential in an area. Unfortunately, these results cannot be used to evaluate the radon concentration inside a building since every building has individual properties (e.g. building fabric, tightness), which can influence the radon concentration. Moreover the radon situation in a building is underlying several influences of meteorological or residents' origin that overlap each other. To get an overview of the radon situation, it is necessary to determine the radon concentration over a longer time, common measurement times are ranging between three months (preferably in the heating period) and a year.

With a better understanding of the radon situation inside a building, it could be possible to get reliable data by a much shorter measurement time. A possible approach is the radon entry rate. The aim of this project was to enhance an existing method for the continuous determination of this parameter, test it respectively to its usability and to analyse possible influencing parameters.

\section{Approach}

The calculation of the radon emission rate is based on a differential equation, which describes the development of the radon concentration in a room [5]:

$$
\begin{aligned}
\frac{d c_{\mathrm{Rn}}(t)}{d t} & =-\lambda \cdot c_{\mathrm{Rn}}(t)+Q_{\mathrm{Rn}, \mathrm{V}}(t)+k(t) \cdot c_{\mathrm{Rn}, a} \\
& -k(t) \cdot c_{\mathrm{Rn}}(t)
\end{aligned}
$$

This includes the radioactive decay of radon-222 $(\lambda)$, the volume-specific radon emission rate $\left(Q_{\mathrm{Rn}, \mathrm{V}}\right)$, the air change rate $(k)$ which expresses the air exchange of a room per hour, the radon concentration outside $\left(c_{\mathrm{Rn}, a}\right)$ and the radon concentration inside a room $\left(c_{\mathrm{Rn}}\right)$. The radon emission rate is defined as a sum of sources, e.g. the radon from the ground and the used building materials. The radon concentration outside was, compared to the absolute changes of the concentration inside, assumed as constant.

For further examinations, the period under observation is minimized so air change rate and radon emission rate can be assumed to be constant. As a result, the time-dependency of the radon emission rate and the air change rate can be omitted:

$$
\begin{aligned}
\frac{d c_{\mathrm{Rn}}(t)}{d t} & =-\lambda \cdot c_{\mathrm{Rn}}(t)+Q_{\mathrm{Rn}, \mathrm{V}}+k \cdot c_{\mathrm{Rn}, a} \\
& -k \cdot c_{\mathrm{Rn}}(t)
\end{aligned}
$$

After solving this differential equation with the initial condition $c_{\mathrm{Rn}}(0)$ the result is:

$$
\begin{aligned}
c_{\mathrm{Rn}}(t)=e^{-(k+\lambda) \cdot t} & \cdot\left(c_{\mathrm{Rn}}(0)-\frac{Q_{\mathrm{Rn}, \mathrm{V}}+c_{\mathrm{Rn}, a} \cdot k}{\lambda+k}\right) \\
& +\frac{Q_{\mathrm{Rn}, \mathrm{V}}+c_{\mathrm{Rn}, a} \cdot k}{\lambda+k}
\end{aligned}
$$

air change rate are known and constant over the period $t=0$ to $t$. If those parameters are thought to be time-dependent the observed period has to be reduced to an interval $\Delta t$. Even if those periods are examined separately from each other, the assumption of a constant radon emission rate and air change rate persists within $\Delta t$. The starting value $c_{\mathrm{Rn}}(0)$ becomes the value of the radon concentration measured before the current value. With known $\Delta t$, the time between two consecutive radon measurements, the course of the radon concentration $c(n)$ can be calculated using the following formula:

$$
\begin{aligned}
c_{\mathrm{Rn}}(n)=e^{-(k(n)+\lambda) \cdot \Delta t} & \cdot\left(\begin{array}{l}
c_{\mathrm{Rn}}(n-1) \\
-\frac{Q_{\mathrm{Rn}, \mathrm{V}}(n)+c_{\mathrm{Rn}, a} \cdot k(n)}{\lambda+k(n)}
\end{array}\right) \\
+ & \frac{Q_{\mathrm{Rn}, \mathrm{V}}(n)+c_{\mathrm{Rn}, a} \cdot k(n)}{\lambda+k(n)}
\end{aligned}
$$

The conversion of the equation for $Q_{\mathrm{Rn}, \mathrm{V}}$ requires several transformations and results in Eq. (5):

$$
\begin{aligned}
& -c_{\mathrm{Rn}}(n-1) \cdot(k(n)+\lambda) \\
& +c_{\mathrm{Rn}}(n) \cdot(k(n)+\lambda) \cdot e^{(k(n)+\lambda) \Delta t} \\
Q_{\mathrm{Rn}, \mathrm{V}}(n)= & \frac{-c_{\mathrm{Rn}, a} \cdot k(n) \cdot\left(e^{(k(n)+\lambda) \Delta t}-1\right)}{e^{(k(n)+\lambda) \Delta t}-1}
\end{aligned}
$$

This equation allows the calculation of a time-dependent radon emission rate. The result is a value which expresses the released activity per cubic metre and hour (volume specific radon emission rate) for each observation at $n$. By multiplying this with the volume of the observed room, the result describes the released activity per hour which is designated as $Q_{\mathrm{Rn}}$.

When defining special boundary conditions ( $t \rightarrow \infty, k$ and $Q_{\mathrm{Rn}, \mathrm{V}}=$ const.) for Eq. (4) the result is:

$$
c_{\mathrm{Rn}}(\infty)=\frac{Q_{\mathrm{Rn}, \mathrm{V}+c_{\mathrm{Rn}, a}} \cdot k}{\lambda+k}
$$

with the above-mentioned conditions, it is now possible to estimate the saturation level of the radon concentration.

Since the real air change rate is assumed to vary over time it has to be measured. This can be achieved by using a tracer gas method. These methods are described by a guideline of the Association of German Engineers (VDI) [6] or in DIN EN ISO 12569:2012. Within this project the concentration decay method was used. A non-radioactive gas is injected into a room and the decrease of the concentration is measured. The air change rate can be determined by using the following equation:

$$
k=\frac{\ln \left(\frac{c_{n-1}}{c_{n}}\right)}{\Delta t}
$$

The measured tracer gas concentration is represented by $c_{n-1}$ and $c_{n}$ while $\Delta t$ identifies the time between those two values. When determining the air change rate by this method, it has to be considEquation (3) allows to determine radon con-
centrations when the radon emission rate and the 
ered that in cases of use of natural occurring gases as a tracer a natural underground concentration exists. This concentration has to be measured and subtracted from the recorded values.

For testing purposes carbon dioxide $\left(\mathrm{CO}_{2}\right)$ was used in this project despite the fact it has several disadvantages. At first, this gas has a natural background that is varying between 400 and $450 \mathrm{ppm}$. The second disadvantageous property is that $\mathrm{CO}_{2}$ is exhaled by humans, so its use in dwellings in the presence of the residents is contradicted. Furthermore, in cases where the concentration of $\mathrm{CO}_{2}$ converges against the natural background the air change rate cannot be determined correctly and the uncertainties will become too large. To avoid this effect a lower threshold value of $900 \mathrm{ppm}$ was defined. When the threshold concentration is reached $\mathrm{CO}_{2}$ has to be injected again. Another property that has to be considered for all gases is the time the tracer gas needs to reach a homogenous concentration in the whole room. The presented disadvantages may disqualify $\mathrm{CO}_{2}$ as a tracer gas for practical use. Alternatives can be found in sulphur hexafluoride $\left(\mathrm{SF}_{6}\right)$ or nitrous oxide $\left(\mathrm{N}_{2} \mathrm{O}\right)$ which are well known for the determination of the air change rate but require complex measurement devices. The decision to use $\mathrm{CO}_{2}$ was based on the availability of cost-effective devices that allow a continuous measurement and the fact that the findings can be transferred easily to the use of the other tracer gases.

Figure 1 shows a section of a measurement series with the detected $\mathrm{CO}_{2}$ concentration and the via Eq. (7) calculated air change rate of a room. The sharp increase of the $\mathrm{CO}_{2}$ concentration was caused by an injection of the tracer gas. Sectors where the air change rate could not be determined due to the homogenization processor the convergence against the natural background concentration were marked as grey bars. In these areas, the ACH was interpolated linearly to get a continuous series of measurement values, which is important for the next step in the calculation process.

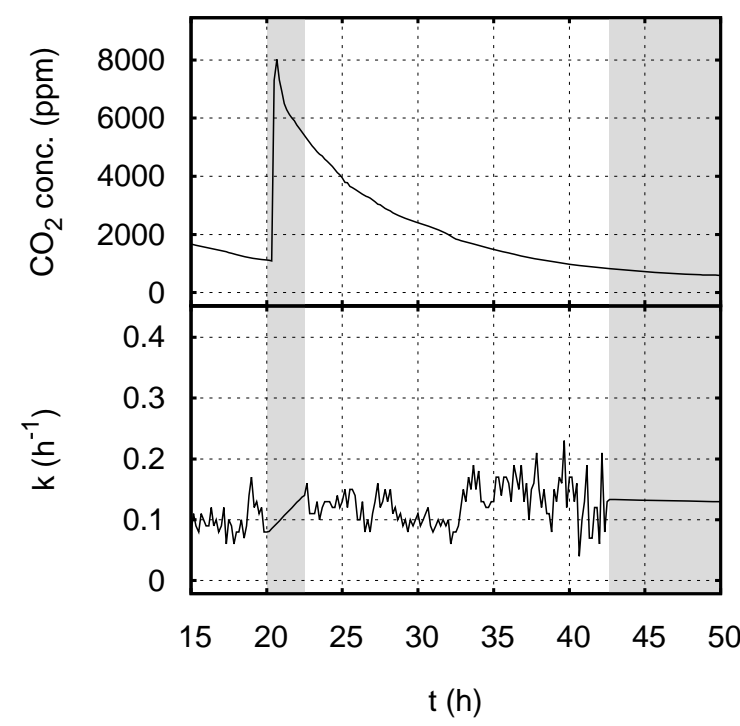

Fig. 1. Section of a measurement series. The decrease of the $\mathrm{CO}_{2}$ concentration can be used for the determination of the air change rate except of the grey marked areas.
As described the radon emission rate can be calculated with the $\mathrm{ACH}$ and the measured radon concentration via Eq. (5). The results show an extremely unsteady signal, which makes it impossible to provide a clear statement on the trend of the radon emission rate. Several attempts have been made to improve the outcome. Radon concentration as well as air change rate were smoothed using the arithmetic and moving average, which showed much better results. A further improvement occurred by calculating the arithmetic or moving average of the results of Eq. (5). Nevertheless, occasionally appearing in sections with strong changes of the air change rate (see Fig. 3) the radon emission rate could not be determined correctly. A promising approach was the use of window functions that have their origin in the digital signal processing. The application of window functions and the positive effects on noisy signals are known due to a former project [5] and delivered useful results.

To apply a window as filter the radon emission rate is calculated by Eq. (5) in the initial step using the corresponding data. After that, a fast Fourier transform (FFT) is performed and the received values are multiplied by the window function. In a last step, the data are transformed back by an inverse Fourier transform (iFFT). This procedure filters the original signal and reproduces the trend of the radon emission rate.

Several of the existing window functions have been tested for their usability and showed less satisfying results. Therefore a modification of a known to be well-performing function was developed: with little changes, a function in the style of the Hamming window $[7,8]$ was created. The general form of the window is presented in Eq. (8). The Hamming window uses the factor $a=2$, when examined in the time domain it shows a similar trend as a Gaussian function. Values in the middle of the window with the width $N$ are weighted stronger. The version used within this project works with the factor $a=1$ and alters the way of weighting. The overall trend is similar to a sigmoid function resulting in stronger weighting of the values at the end of the window. Both versions of the function are working as a low-pass filter.

$$
w(n)=0.54-0.46 \cdot\left(\frac{a \cdot \pi \cdot n}{N-1}\right)
$$

Satisfying results with this modified window function were obtained using a window width of $N=128$. This seemed to be the best compromise between noise reduction through filtering and the precision of the reconstruction based on the original signal.

\section{Measurement devices}

AlphaGUARDs from Saphymo GmbH (former Genitron) were used for the radon measurements while the $\mathrm{CO}_{2}$ concentration and room climate parameters were determined with CDL210 by WöhlerMessgeräteKehrgeräte $\mathrm{GmbH}$. All devices ran with a log 
rate for $10 \mathrm{~min}$. Meteorological parameters were sampled by a nearby weather station of the Hessian Agency for the Environment and Geology (HLUG).

\section{Results}

Before the first application of the described method the procedure was tested using simulated data and measurement data from a former project. The simulated data of the radon concentration were generated by a program which uses defined parameters (e.g. room volume, air change rate, radon background concentration and radon source) and known uncertainties of the measurement devices. These radon concentrations and the predefined air change rates were used to reconstruct the radon emission rate.

An example is presented in Fig. 2. While the trends of the defined and the reconstructed radon emission rate are plotted in the lower part the air change rate is shown in the middle. The result of the simulation is the radon concentration and can be seen in the upper part. The changes in the radon concentration were induced by an increase or decrease of the radon source and the air change rate. The reconstructed emission rate (modified window function, $N=128$ ) was compared to the predefined radon source and showed good correspondence. Especially within constant parts of the radon source, a better conformity with the predefined values was achieved as shown in Fig. 2. The final rise of the radon emission is induced by a sharp increase of the radon source that is not shown in this graph. The reconstruction lacks precision in areas of sharp

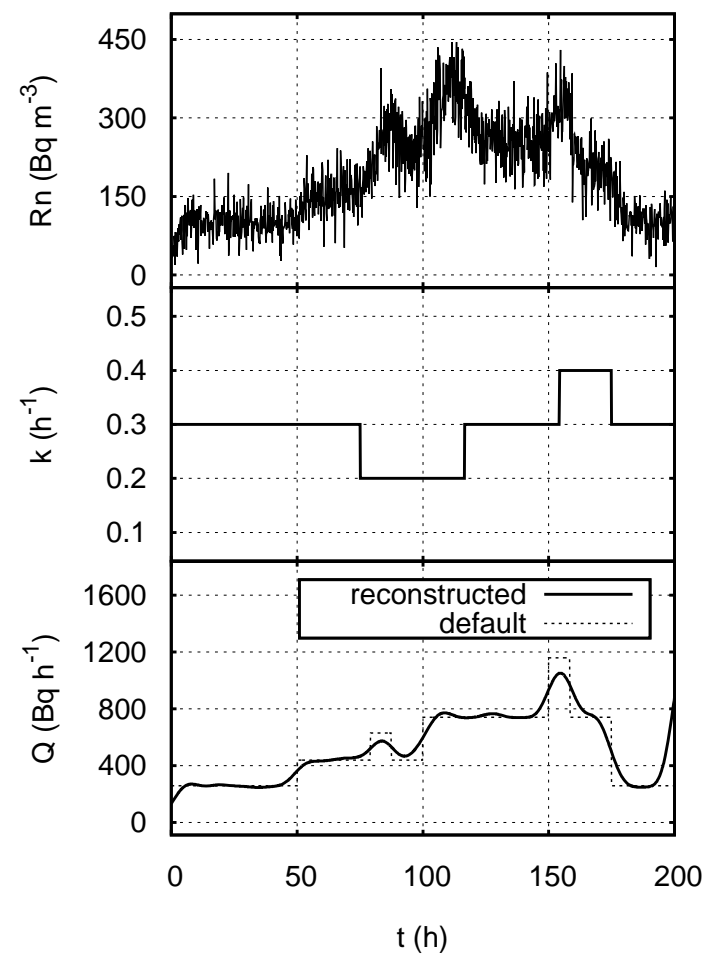

Fig. 2. Example of a simulation. Room volume, air change rate and radon source were defined before simulating the radon concentration. The simulation result and the predefined air change rate were used to reconstruct the radon source.

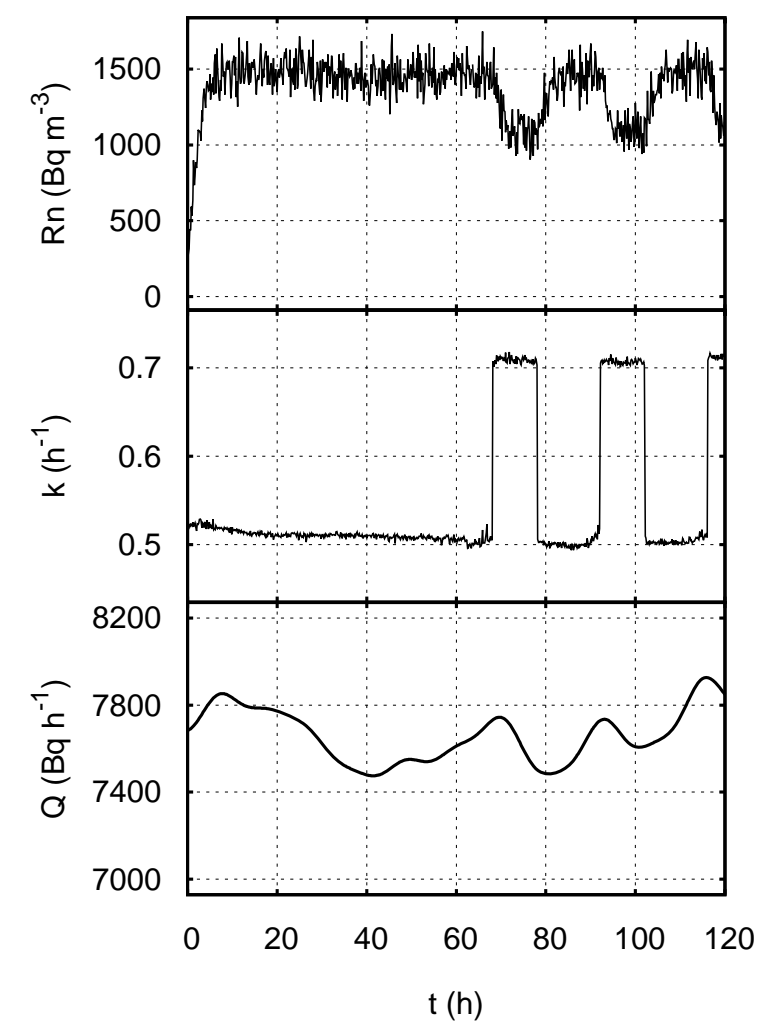

Fig. 3. Example of measurement taken inside an climate chamber. The radon concentration was produced by pitchblende, the air change rate $(\mathrm{ACH})$ could be changed utilizing a fan. The variation of the radon emission rate is caused by the abrupt changes of the $\mathrm{ACH}$ which cannot be ruled out by the calculation process.

edges resulting from strong changes of the $\mathrm{ACH}$ or the emission rate. Despite this, the results were considered sufficient for the application in real objects since the air change rate and radon emission rate are assumed not to follow a discontinuous function. The shown result is the best compromise between sufficient filtering and information loss.

Besides the simulations, data from measurements of a former project were used to verify the method. These measurements have been performed in a climate chamber with a volume of $10 \mathrm{~m}^{3}$. Several radon sources (e.g. pitchblende and soil samples with high radium content) were placed inside the chamber to produce radon concentrations with different levels. The air change rate of the chamber was defined by an installed fan, which produced a gauge pressure. With the known flow rate and the chamber volume the air change rate could be determined. A measurement series can be seen in Fig. 3. The measured radon concentration is plotted in the upper part, the graph below shows air change rate, the calculated radon emission rate is represented by the plot in the lower part. The used radon source can be assumed to be constant, the reconstructed radon emission rate has a mean value of $7660 \mathrm{~Bq} \cdot \mathrm{h}^{-1}$ and a margin of deviation of $\pm 3 \%$. The peaks around $t=70,90$ and $115 \mathrm{~h}$ are caused by abrupt changes of the air change rate and could not be ruled out by the calculation process. These variations do not represent the actual trend of the radon emission rate in this part of the measurement series. 
After verifying the possible usability of the method by numerous simulations and with data from older measurements, an uninhabited building used as storage room was used for further tests under real conditions. It consists of two connected rooms and an attic. Each room has one window; the attic is partially open and was used as a weather-proof measurement point of the outdoor conditions. One radon measurement device and one room climate measurement device were put into each room and the attic. The parameters were recorded over a period of eight weeks (end of October 2014 to the middle of December 2014). Besides gaining experience with the method to determine the air change rate different measurement conditions were arranged (e.g. opening or closing the door between the two rooms and opening of the windows). Due to the rather low measured radon concentrations (up to $200 \mathrm{~Bq} \cdot \mathrm{m}^{-3}$ ) an additional strong radon source was placed into the building for a short period of time to imitate a building with a high radon entry rate. The expected increase of the radon entry rate was detected based on the calculations via Eq. (5) and application of the window function.

A major problem during the measurements was to provide a $\mathrm{CO}_{2}$ concentration over the whole recording time that can be used for the determination of the air change rate. Because this was not possible to achieve there are several gaps in the calculated air change rate data. Since it is not possible to reconstruct the radon emission rate when air change rate data are missing gaps were linearly interpolated. To avoid wrong interpretations during the analysis produced by the interpolation the gaps are marked with grey bars in all diagrams.

The radon emission rate of the investigated object has a mean value $Q_{\mathrm{Rn}}=715 \mathrm{~Bq} \cdot \mathrm{h}^{-1}$, the deviation was up to $30 \%$. Figure 4 shows a part of a measurement series. In addition, the difference between the inside and outside temperature of the building is plotted. With every increase of the temperature difference

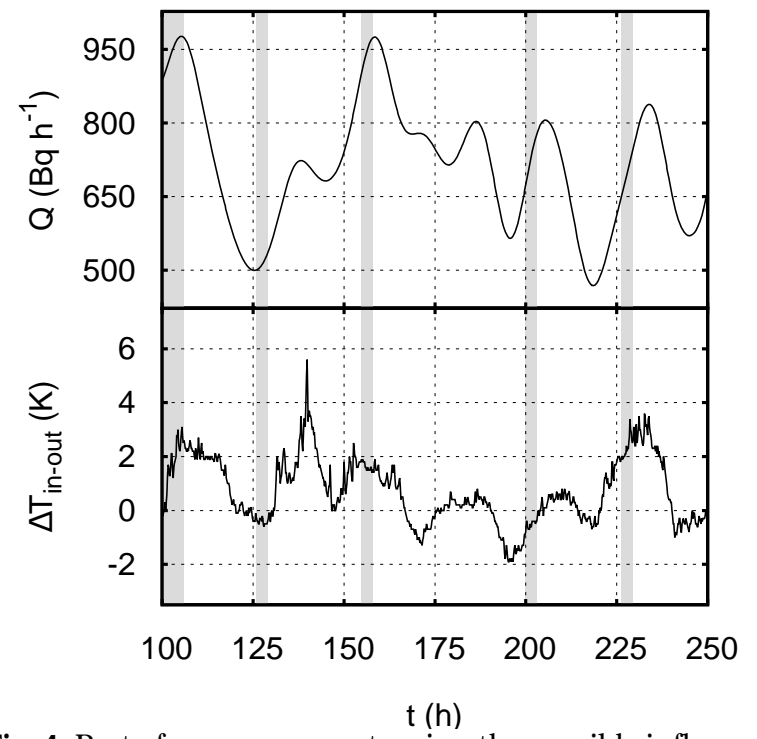

Fig. 4. Part of a measurement series, the possible influence of the temperature difference between inside and outside of the building can be seen. Gaps with interpolated air change rate are marked with grey bars. an increase of the radon emission rate occurred, a similar behaviour that has been observed in [9] based on measurements of the radon concentration. A possible explanation of this effect could be that warm air from inside escaped through leaks in the attic and produced a negative pressure which forced the radon to exhale from the ground. The analysis of the correlation coefficient showed results between 0.3 and 0.9 . Other parameters like air pressure and humidity showed no significant correlation.

\section{Discussion}

The method for the determination of the radon emission rate was verified by simulations and measurements. Nevertheless, there is potential for improvements. When determining the air change rate with $\mathrm{CO}_{2}$ as tracer gas in uninhabited rooms it is recommended to use a constant injection method to prevent the concentration from decreasing to the background concentration. By now a device for the constant injection of $\mathrm{CO}_{2}$ has been obtained, and the first results show that the air change rate can be calculated correctly on a continuous base over long periods of time. On a long-term view more suitable gases like $\mathrm{SF}_{6}$ or $\mathrm{N}_{2} \mathrm{O}$ should be used when determining the radon emission rate in dwellings while they are inhabited.

As consequence of the continuous determination of the air change rate, the analysis of possible influencing parameters of the radon emission rate can be done based on a bigger database. In addition, other possible influencing parameters will be considered, for example the pressure difference between inside and outside.

Further long-time measurements have to show if the radon entry rate remains on a constant level or if possible variations, caused by existing influences, can be corrected. Depending on the results of this analysis it has to be evaluated if short-time measurements provide the same information about the radon situation inside a building as long-time measurements.

Acknowledgment. The work was funded by the Ministry of the Environment, Climate Protection, Agriculture and Consumer Protection of Hesse (HMUKLV) as part of the project HMU32206031. We also would like to thank the Hessian Agency for the Environment and Geology (HLUG) for providing meteorological data.

The work was performed in Giessen (Bundesland Hessen) in Germany.

\section{References}

1. Grosche, B., Kreuzer, M., Kreisheimer, M., Schnelzer, M., \& Tschense, A. (2006). Lung cancer risk among German male uranium miners: a cohort study, 1946-1998. Br. J. Cancer, 95, 1280-1287.

2. Darby, S., Hill, D., Deo, H., Auvinen, A., Barros-Dios, J. M., Baysson, H., Bochicchio, F., Falk, R., Farchi, 
S., Figueiras, A., Hakama, M., Heid, I., Hunter, N., Kreienbrock, L., Kreuzer, M., Lagarde, F., Mäkeläinen, I., Muirhead, C., Oberaigner, W., Pershagen, G., Ruosteenoja, E., Schaffrath Rosario, A., Tirmarche, M., Tomášek, L., Whitley, E., Wichmann, H. -E., \& Doll, R. (2006). Residential radon and lung cancer-detailed results of a collaborative analysis of individual data on 7148 persons with lung cancer and 14208 persons without lung cancer from 13 epidemiologic studies in Europe. Scand. J. Work Environ. Health, 32(Suppl. 1), $1-84$

3. Kendall, G. M., \& Smith, T. J. (2002). Doses to organs and tissues from radon and its decay. J. Radiol. Prot., $22,389-406$.

4. Council of the European Union. (2014). Council Directive 2013/59/EURATOM of 5 December 2013 laying down basic safety standards for protection against the dangers arising from exposure to ionising radiation. Brussels: O. J. EU.
5. Roessler, F. A., Azzam Jai, T., Grimm, V., Hingmann, H., Orovwighose, T., Jach, N., \& Breckow, J. (2014). First steps in the development of a possible measurement method to estimate the radon concentration as an indicator of the indoor air quality. Nucl. Technol. Radiat., 29(Suppl.), 52-58.

6. VDI-Association of German Engineers. (2001) Measurement of the indoor air change rate. Duesseldorf: Association of German Engineers (VDI). (VDI 4300-7).

7. Blackman, R. B., \& Tukey, J. W. (1958). Measurement of power spectra. New York: Dover Publications, Inc. 8. Smith, S. (1999). The scientist and engineer's guide to digital signal processing. San Diego: California Technical Publishing.

9. Udovicic, V., Filipovic, J., Dragic, A., Banjanac, R., Joković, D., Maletić, D., Grabež, B., \& Veselinović, N. (2014). Daily and seasonal radon variability in the underground low-background laboratory in Belgrade, Serbia. Radiat. Prot. Dosim., 160(1/3), 62-64. 